\title{
CONCEPTOS EPISTEMOLÓGICOS CLÁSICOS: SU UTILIDAD EN LA CONSTRUCCIÓN DE TESIS Y DISERTACIONES EN LAS CIENCIAS SOCIALES Y HUMANAS
}

\section{Classical epistemological concepts: their application in the construction of theses and dissertations in social and human sciences.}

\section{Jorge Santos ${ }^{1}$}

\begin{abstract}
RESUMEN
Este artículo aborda algunos conceptos principales de grandes las discusiones epistemológicas que acontecieron durante el siglo XX. En el círculo de Viena, en la doctrina falsacionista de Popper, y en la Nueva Filosofía de la Ciencia se desarrollan sucesivamente interesantes discusiones y polémicas en torno a la naturaleza del conocimiento científico, su método para probar hipótesis, los criterios para distinguir el conocimiento científico del que no lo es, las diferencias o similitudes entre ciencias naturales y sociales, etc. En estas discusiones se proponen diversas perspectivas y conceptos epistemológicos para intentar responder las preguntas planteadas en torno al conocimiento científico. Estas polémicas tienen un alto nivel teórico y suelen ser abordadas en cursos de epistemología, metodología o filosofía de las ciencias. En este trabajo pretendemos abordar algunos de estos conceptos desde una perspectiva novedosa, atendiendo no solamente a su valor teórico o explicativo, sino especialmente a su utilidad pragmática al momento de construir una disertación de maestría o una tesis de doctorado en el área de las ciencias sociales y humanas. A este fin el artículo selecciona algunos de los conceptos clásicos esgrimidos en las discusiones señaladas y los aborda a partir de la perspectiva propuesta, es decir atendiendo y señalando la utilidad práctica de estos conceptos al momento de la construcción de una tesis o disertación. Particularmente los conceptos seleccionados son los de: razonamiento, problema, hipótesis, prueba y división de escuelas en el ámbito de las ciencias humanas y sociales.
\end{abstract}

Palabras clave: Epistemología. Ciencias sociales. Ciencias humanas. Razonamiento. Hipótesis.

\footnotetext{
ABSTRACT

This article addresses some of the main concepts of the epistemological discussions that occurred during the 20th century. In the Vienna circle, in Popper's falsificationist doctrine, and in the New Philosophy of Science, interesting discussions and controversies develop successively about the nature of

${ }^{1}$ CONICET/Universidad Nacional de Hurlingham.

E-mail: jorgesantosuba@gmail.com. ORCID: 0000-0002-9081-5881.
} 
scientific knowledge, its method to test hypotheses, the criteria to distinguish scientific knowledge of which it is not, the differences or similarities between natural and social sciences, etc. In these discussions, various perspectives and epistemological concepts are proposed to try to answer the questions asked about scientific knowledge. These controversies have a high theoretical level and are usually addressed in epistemology, methodology or philosophy of science courses. In this work we intend to approach some of these concepts from a novel perspective, attending not only to their theoretical or explanatory value, but especially to their pragmatic usefulness when constructing a master's thesis or a doctoral thesis in the area of social sciences and human. With this aim in mind, the article selects some of the classic concepts used in the indicated discussions and approaches them from the proposed perspective, that is, attending to and pointing out the practical utility of these concepts at the time of the construction of a thesis. Particularly the selected concepts are those of: reasoning, problem, hypothesis, test, and division of schools in the field of human and social sciences.

Keywords: Epistemology. Social sciences. Human sciences. Reasoning. Hypotheses.

\section{Introducción}

Este artículo aborda algunos conceptos más relevantes propuestos en las importantes discusiones epistemológicas que acontecieron durante el siglo XX. Durante el siglo pasado en el círculo de Viena, en la doctrina falsacionista de Popper, y en la Nueva Filosofía de la Ciencia se desarrollan sucesivamente interesantes discusiones y polémicas en torno a la naturaleza del conocimiento científico, su método para probar hipótesis, los criterios para distinguir el conocimiento científico del que no lo es, las diferencias o similitudes entre ciencias naturales y sociales, etc. En estas discusiones se proponen diversas perspectivas y conceptos epistemológicos para intentar responder las preguntas planteadas en torno al conocimiento científico. En este trabajo pretendemos abordar algunos de estos conceptos desde una perspectiva novedosa, atendiendo no solamente a su valor teórico o explicativo, sino especialmente a su utilidad pragmática al momento de construir una disertación de maestría o una tesis de doctorado en el área de las ciencias sociales y humanas. Es común en los distintos niveles de educación superior tener materias ligadas al conocimiento científico, epistemología o metodología de las ciencias. Sin embargo, la tarea de investigación concreta suele aprenderse en la práctica científica con la guía de un orientador y no 
siempre se conecta con los conceptos teóricos aprendidos en las clases citadas. Suele existir una desconexión entre las reflexiones y discusiones teóricas en el terreno de la epistemología y la práctica científica concreta que suele iniciarse en los niveles de maestría y doctorado.

Este artículo entonces más que ocuparse de discusiones epistemológicas referidas a la naturaleza de la ciencia, o lo adecuado de este o aquel criterio para diferenciar el conocimiento científico de otras formas de conocimiento, o de la corrección o incorreción de las diferentes metodologías, tendrá una finalidad pragmática: presentar algunos conceptos epistemológicos clásicos, abstrayéndose de las polémicas teóricas respecto a ellos, en función de su aplicación práctica. Es decir, se ocupará no solo del valor teórico y explicativo de los conceptos epistemológicos, sino de la utilidad de esos conceptos respecto a la comprensión y a la construcción de una tesis o disertación en el área de las ciencias humanas y sociales.

Al momento de presentar un proyecto de tesis a niveles de maestría y doctorado, e incluso, al momento de solicitar becas u otro tipo de financiación para el proyecto, el mismo debe cumplir ciertos requisitos básicos: plantear el problema que se pretende investigar, encuadrarlo en alguna de las áreas de conocimiento, plantear una hipótesis dentro de un marco teórico adecuado y una metodología para investigarla a fin de confirmarla o no. Estos requisitos muestran implícitamente una concepción del conocimiento científico estructurada a partir de conceptos estudiados en el área de la epistemología. Por lo tanto, estos conceptos además de su valor teórico tienen un valor práctico o pragmático pues son útiles para la construcción de un buen proyecto de investigación que, en tanto permite acceder al reconocimiento de las agencias de investigación, resulta ser una actividad fundamental para el investigador científico. Este valor pragmático es menos estudiado que su valor teórico, esto se puede percibir fácilmente cuando se abordan las principales discusiones del área de la epistemología. El objetivo de este artículo entonces es explorar el valor pragmático de algunos conceptos epistemológicos en tanto sirven para comprender la tarea de investigación de un científico social, así como soporte para la construcción de sus tesis y disertaciones. 
Atendiendo a esta finalidad el artículo comienza con la explicitación del marco teórico y metodológico, luego expondrá en diferentes apartados los conceptos seleccionados a fin de explicitar su utilidad práctica en la construcción de tesis y disertaciones en el área de las ciencias sociales y humanas.

\section{Marco teórico y metodológico}

El método que se propone para abordar esta temática en el analítico en el sentido en que se propondrán algunos conceptos epistemológicos clásicos, se los definirá y se analizará la definición en sus partes o sentidos y términos más pequeños o específicos que lo componen (BLASCO, 1996). Sin embargo, el método analítico tendrá una variación, porque el análisis de estos estará en función de su utilidad práctica para la construcción de una tesis o disertación especialmente en el ámbito de las ciencias sociales y humanas. Por lo tanto, podemos hablar de un método analítico-pragmático en el sentido de que el análisis de los conceptos no se limitará a sus aspectos puramente teóricos-semánticos, sino que se extenderá a su utilidad práctica para el científico social.

Con respecto al marco teórico, es decir los autores dentro del campo de la epistemología que enuncian los conceptos seleccionados serán algunos de los clásicos del área: a partir de Copi (2013) abordaremos el concepto lógico de razonamiento, con Hempel (1983) y Popper (1980) la noción fundamental de problema científico e hipótesis, de Tomas Kuhn (2004) tomaremos la idea de fragmentación en escuelas de las ciencias sociales, concepto que permite comprender la necesidad de construcciones de marcos teóricos y metodológicos específicos. Además, recurriremos a autores que abordan el pensamiento de los autores citados como Klimovsky (2005), Gaeta y Gentile (2007)

Una cuestión importante en este apartado de acuerdo con la metodología postulada es aclarar el sentido del concepto de "ciencias humanas y sociales" que estamos utilizando. Veremos que esta necesidad constante de aclarar nuestros conceptos, definirnos de acuerdo de acuerdo con el sentido específico con que lo queremos utilizar y en referencia a nuestro marco te- 
órico, es una de las características de la investigación social y humana que la diferencia de otras áreas de conocimiento. La definición de "ciencias humanas y sociales" no es sencilla pues se trata de un concepto problemático y en disputa entre diferentes posiciones teóricas al respecto. En un sentido muy amplio y casi trivial podemos decir que "humanas" son las ciencias que estudian al ser humano y su relación con la sociedad (BUENO, 1978). También podemos afirmar con Luque (2010) de manera más específica que a mediados del siglo diecinueve surge el interés práctico por resolver problemas económicos, sociales y políticos propios del orden capitalista. Entonces la pregunta científica se orienta hacia el hombre, así la necesidad de dominar y controlar los fenómenos sociales transforma al hombre en el objeto de estudio de este nuevo dominio de saber. Estas disciplinas recortan desde distintas perspectivas, sus objetos de estudio en relación con la actividad del hombre.

Pero estos sentidos son todavía muy generales e incluso el carácter científico de las disciplinas agrupadas bajo el nombre de "humanas y sociales" no siempre es reconocido por todos los epistemólogos. Incluso algunas disciplinas incluidas en el área de "humanidades" no reclaman el adjetivo "científico" para sí mismas, como ocurre con varias corrientes filosóficas. $\mathrm{La}$ alternativa que tomamos entonces para especificar este sentido general será un recurso a la práctica, al uso pragmático de ese término por las agencias científicas. En términos técnicos vamos a recurrir a una definición más extensional o 'por extensión' es decir “enumerar los miembros de un conjunto" (Russell, 1956, p. 88), que a una estrategia intencional o 'por comprensión' es decir aquella "que pone de manifiesto una propiedad definidora" (Russell, 1956, p. 88). El conjunto de las disciplinas que nombramos como "ciencias humanas y sociales" se corresponde de manera bastante precisa con las incluidas en las grandes áreas de conocimiento de las agencias de investigación científica bajo cientos títulos. En el caso de CONICET en Argentina la referencia se corresponde con las disciplinas incluidas bajo el título "Ciencias Sociales y Humanidades". En el caso de CAPES en Brasil esta referencia se agrupan bajo dos títulos "Ciencias Humanas" y

\footnotetext{
${ }^{2}$ https://www.conicet.gov.ar/definiciones-tecnicas/.
} 
"Ciencias Sociales Aplicadas"3. Si bien estos conjuntos no se corresponden exactamente, las diferencias son pequeñas $\mathrm{y}$, atendiendo al carácter extensional con el que optamos por definir nuestra referencia, podemos afirmar que el sentido se corresponde con la unión de estos conjuntos de disciplinas. Es una manera pragmática y relativamente simple de resolver un problema teóricamente complejo y pone en evidencia el objetivo de este artículo y la estrategia metodológica seleccionada.

En los apartados siguientes entonces abordaremos conceptos importantes de la epistemología ligados a los trabajos de los autores citados para referirnos a su utilidad en el trabajo de construcción de una tesis o disertación. Comenzaremos con la idea de razonamiento, seguiremos con el concepto de problema científico e hipótesis y finalmente abordaremos la construcción de marco teórico en relación con la fragmentación de escuelas en las ciencias sociales postulada por Kuhn. En el apartado final sacaremos conclusiones.

\section{Construir una disertación como un razonamiento o argumento complejo}

Uno de los conceptos importantes para la filosofía clásica de las ciencias es el de razonamiento. Las discusiones epistemológicas iniciadas durante principios el siglo veinte en el Círculo de Viena dan un papel muy relevante a la función de la lógica y de los distintos tipos de razonamientos al momento de analizar el procedimiento de la ciencia para obtener y probar el conocimiento. Se discute sobre el papel de los razonamientos deductivos e inductivos y de las diferentes metodologías propuestas en torno a ellos, esta polémica toma especial intensidad a partir del planteo falsacionista de Popper.

Sin embargo, el objetivo de este análisis no es abordar esas discusiones en sus detalles, sino definir, de acuerdo con lo establecido en el marco metodológico, el concepto en su acepción más general y aceptada a fin de analizar su utilidad en la construcción de una tesis en el área de las ciencias humanas y sociales.

\footnotetext{
${ }^{3}$ https://capes.gov.br/avaliacao/sobre-as-areas-de-avaliacao.
} 
Gianella (2002) nos dice que un razonamiento es un conjunto de proposiciones $^{4}$ o enunciados en el que una de ellas, llamada conclusión, se pretende fundada en, o se infiere de, otras que se llaman premisas.

Razonamiento" suele entenderse como sinónimo de "argumento", así Copi señala que:

\begin{abstract}
un argumento es un grupo de proposiciones del cual se dice que una de ellas se sigue de las otras, consideradas como base o fundamento de la verdad de esta (...) Para que pueda decirse que existe un argumento, tiene que haber una estructura en ese conjunto de proposiciones, una estructura que capture o muestre alguna inferencia. Esta estructura se describe usando las palabras premisas y conclusión. La conclusión de un argumento es una proposición es que se afirma con base en otras proposiciones del argumento. Estas otras proposiciones, las cuales se afirma (o asume) que son soportes de la conclusión, son las premisas del argumento. (Copi et al, 2013, p.7)
\end{abstract}

¿Cuál es la utilidad de este concepto para la construcción de una tesis o disertación? Lo que esta idea puede aportar es significativo si entendemos que la construcción de una tesis o disertación consiste en construir un razonamiento o argumento complejo. Toda tesis o disertación incluye una conclusión o varias conclusiones, en general de manera explícita pues el último capítulo suele ser justamente el de las conclusiones o consideraciones finales. Los capítulos de la tesis en general son premisas en el sentido de que, en una tesis bien estructurada, su función es fundamentar, sostener o probar la conclusión que, en general, aparece en el capítulo final. Al momento de evaluar una tesis, el análisis de la coherencia interna es crucial, la primera lectura tiene que ver con si hay coherencia lógica entre lo propuesto en la introducción y el resultado expuesto en la conclusión, así como la consistencia del resultado final con lo desarrollado en los capítulos previos.

Claro que la envergadura y extensión de una tesis de posgrado hacen se trate de un razonamiento sumamente complejo en general compuesto por varios razonamientos intermedios. En general cada capítulo de una tesis implica un razonamiento en sí mismo con una conclusión parcial que servirá luego como argumento para fundamentar la conclusión final o resultado de la investigación. Esto no es problemático, al contrario, es correcto afirmar que ninguna afirmación es en sí misma premisa o conclusión, por lo que la

\footnotetext{
${ }^{4}$ Proposición entendida como una oración con sentido informativo a la que se puede atribuir valor de verdad (verdadera o falsa).
} 
conclusión de un razonamiento puede perfectamente ser premisa de otro, esta concatenación da coherencia interna a un trabajo.

Además, en el ámbito de las ciencias humanas y sociales hay que sumar otra complejidad: los razonamientos suelen sustentarse en metodologías diversas que precisan se explicitadas en la propia tesis por ejemplo metodología analítica, hermenéutica, dialéctica, fenomenológica, análisis de discurso, etc. Esto suma complejidad en cuanto a la "lógica" propia de cada metodología que no se corresponde necesaria y estrictamente con la corrección de la llamada "lógica formal" o al análisis estrictamente deductivo de los razonamientos. Esto no invalida la afirmación de que con esas metodologías se construyen argumentos o razonamientos complejos para probar lo que será la conclusión de la tesis. Una cuestión diferente es el estándar con el que evaluamos estos argumentos, si lo hacemos a partir de una lógica formal estricta o a partir de un criterio más amplio y menos estricto como el de las conocidas lógicas paraconsistentes que admiten ciertas tensiones internas en desarrollos teóricos especialmente en el campo jurídico, que desde una perspectiva estricta no serías aceptables. (MIRÓ QUESADA, 1988). En este sentido la idea de razonamiento que utilizamos es definida en sentido amplio, en el sentido de tiene que haber una relación clara de justificación, es decir de fundamentación, entre lo desarrollado en la tesis y las conclusiones a las que se arriban.

Este requisito de orden y coherencia interna puede resultar algo peculiar para los que han transitado el proceso de investigación y construcción de una tesis y sobre todo para los que están transitando actualmente este proceso. Sucede que en la práctica una investigación no suele presentarse tan ordenada como suponemos, muchas veces intuimos la conclusión primero y luego buscamos las pruebas, o al contrario primero aparecen algunas premisas y luego una conclusión, o primero el problema y luego otros aspectos relevantes, o a la inversa. En pocas palabras el proceso de investigación y de construcción de la tesis no parece ser tan lineal y ordenado, no parece suceder de acuerdo con la coherencia que, postulamos, debe tener un argumento bien construido.

Es cierto, el proceso real de una investigación suele ser bastante más caótico y menos lineal y organizado que su producto final: un argumento 
bien construido y expresado que constituye una disertación de maestría o una tesis de doctorado. Pero eso es algo común para todo argumento, Copi señala:

Cuando los psicólogos examinan el proceso de razonamiento lo encuentran sumamente complejo, emocional en alto grado y consistente en desmañados procedimientos de ensayo y error iluminados por repentinos chispazos de comprensión a veces inconexos en apariencia. Ellos son de la mayor importancia para la psicología. Pero estos oscuros caminos por los cuales la mente llega a sus conclusiones durante los procesos reales de razonamiento, no son en absoluto de incumbencia del lógico. Solo le interesa la corrección del proceso una vez terminado. Su problema es siempre el siguiente: la conclusión a la que ha llegado, ¿deriva de las premisas usadas o afirmadas? (COPI, 1992, p.5)

Del mismo modo, parafraseando la cita, el proceso de investigación real y el trabajo del maestrando o doctorando puede ser sumamente complejo, emocional en alto grado, con procedimientos de ensayo y error y chispazos de comprensión a veces inconexos en apariencia. Sin embargo, al momento de escribir la tesis la investigación tendrá que tomar la forma de un argumento comprensible y bien estructurado que fundamente la conclusión a la que se arriba. Es el momento de darle un orden a ese proceso que fue tal vez más asistemático y en apariencia inconexo respecto de su resultado final. Esto no quiere decir que el proceso real de investigación está ausente en el resultado final. Estará presente conectado y articulado lógicamente de tal forma que la conclusión a la que se arriba esté fundamentada en un argumento que pueda ser entendido por quien lo lea. E incluso, en un resultado ideal, que pueda llegar a convencerlo.

Conocer esta lógica del proceso de investigación y construcción del conocimiento en las ciencias sociales es útil para quien se inicia en una investigación. Pues durante este proceso nos sentimos a veces desorientados, no percibimos exactamente el sentido de las lecturas o estudios que realizamos, en otros momentos en cambio tenemos intuiciones de comprensión y entendimiento. No hay que desesperar, son circunstancias que suelen suceder durante toda investigación. Con esa materia se construye el argumento que será el producto final de nuestra investigación: nuestra tesis o disertación. En definitiva, la ciencia se materializa y socializa a través del lenguaje y de las afirmaciones que expresan su objeto de estudio (GAETA, et al, 1990). 
Las afirmaciones que expresan y constituyen el conocimiento científico, es decir sus teorías e hipótesis, se fundan en argumentos y estos argumentos son productos de investigaciones. El argumento como resultado de una investigación necesita tener coherencia y consistencia a fin de ser comprensibles para todos los versados en el área y en particular, compresible y convincente para los especialistas que serán jurados de nuestras tesis.

\section{Definiendo el problema y la hipótesis de una investigación}

Carl Hempel, una de las figuras importantes del Circulo de Viena, realiza un ya clásico análisis sobre el rol de las hipótesis en las investigaciones científicas. El autor además de epistemólogo era físico por lo que presta especial atención a las ciencias naturales. Sin embargo, varios de sus planteos incluyen y son interesantes, para el trabajo científico en el área de las humanidades. Este interés radica por un lado en el valor intrínseco del procedimiento postulado por Hempel que puede ser útil como esquema para plantear una investigación social. Pero también tiene un interés pragmático pues la concepción de ciencia implícita que evidencian las agencias científicas estatales se corresponde en varios aspectos con los conceptos clásicos que, como los del autor, están inspiradas en las ciencias naturales. Para postularse como tesista de maestría o doctorado y más aún, para conseguir becas u otro tipo de financiamiento, se solicita al postulante que explicite el problema a abordar, la hipótesis principal que guiará la investigación y la metodología con la que realizará la investigación y evaluará los resultados de la misma.

La idea de que la ciencia se ejerce a partir de hipótesis entendidas como respuestas a problemas científicos y de su evaluación, en general, a partir de métodos empíricos es la concepción más común y generalizada de entender el procedimiento que utilizan los investigadores científicos (Klimovsky, 2005). Consecuentemente, se corresponde con los requisitos solicitados por las agencias estatales que financian y regulan estas actividades al momento de presentar un proyecto de investigación.

Y esto es válido tanto para el aspirante en el área de ciencias naturales como en el de humanas y sociales. Este es el motivo por el que, más allá 
del valor teórico propio que tienen los conceptos expuestos, es pragmáticamente importante conocerlos y comprenderlos al momento de iniciarse en el trabajo de investigación.

De acuerdo con el método postulado presentaremos los conceptos definidos por Hempel y analizaremos su utilidad para la investigación en el área de humanas y sociales.

Pero cabe la posibilidad de que lo que se nos exija en esa primera fase de la investigación científica sea reunir todos los hechos relevantes. Pero ¿relevantes con respecto a qué? (...) supongamos que la investigación se refiere a un problema específico. ¿Es que no empezaríamos, en ese caso, haciendo acopio de todos los hechos...o, mejor, de todos los datos disponibles que sean relevantes para ese problema? (...) el tipo concreto de datos que haya que reunir no está determinado por el problema que se está estudiando, sino por el intento de respuesta que el investigador trata de darle en forma de conjetura o hipótesis (...) las hipótesis, en cuanto intentos de respuesta, son necesarias para servir de guía a la investigación científica. Esas hipótesis determinan, entre otras cosas, cuál es el tipo de datos que se han de reunir en un momento dado de una investigación científica. (HEMPEL, 1983, pp. 28-29)

En el párrafo señalado se postula la idea de que la investigación científica parte de un problema bien planteado y de un intento de respuesta de este que llamamos hipótesis. Esta hipótesis guía la investigación, pues permite seleccionar los datos relevantes a fin de evaluar si se trata de una respuesta adecuada al problema.

La construcción y definición del problema es, por lo tanto, un paso fundamental para cualquier investigación en cualquier área, incluidas las de nuestro interés. Sin embargo, la presencia de hipótesis como posible respuesta al problema y su función de guía de la investigación no siempre es tan clara o evidente en nuestro ámbito. Muchas veces al principio de una investigación solo tenemos un problema, y este parece ser el que guía le investigación, esto es bastante frecuente en ciencias sociales. Tenemos un problema plateado a partir de ciertos datos o conceptos, pero no necesariamente hipótesis. Si bien esta situación es posiblemente más frecuente, no es una exclusiva de las ciencias sociales. Como el propio Hempel señala siempre que se trata la solución a un problema, tiene que haber implícitamente alguna hipótesis general que establezca los parámetros de la solución. 
Por ejemplo, el objetivo de este artículo puede enunciarse como el siguiente problema: ¿Hay conceptos de la epistemología clásica que sean pragmáticamente útiles para las investigaciones en ciencias humanas y sociales? En caso de respuesta positiva ¿Cuáles? En nuestra respuesta se evidencia la hipótesis de que sí existen conceptos pragmáticamente útiles y enunciamos algunos de los que consideramos más interesantes. Es decir, trabajamos bajo la hipótesis general de que existen al menos tres conceptos interesantes (a esta altura del artículo): razonamiento, problema e hipótesis. Nuestro argumento se construye enunciando el problema u objetivo del artículo, dando una respuesta positiva al mismo a través de esa hipótesis implícita y enunciando e intentando demostrar la utilidad de estos conceptos. Tener un problema bien formulado, hacer buenas preguntas sobre el tema de interés e intentar responderla supone siempre hipótesis implícitas, aunque no sean total y claramente formuladas al inicio de la investigación. Este procedimiento de enunciar problemas, generar hipótesis e intentar justificarlas, sirve para guiar la investigación y estructurar el razonamiento complejo que constituye una tesis de maestría o doctorado.

Hay otras dos cuestiones ligadas a estos conceptos que el autor vienés trabaja, son el surgimiento de las hipótesis y la justificación o prueba de estas. En primer lugar, señala que la respuesta al problema, es decir, el surgimiento de la hipótesis no es un proceso mecánico, al contrario, es producto de la creatividad y la imaginación del científico (HEMPEL, 1983). Creemos que es interesante resaltar esta idea: creatividad, imaginación, surgimiento de nuevas hipótesis, de nuevas perspectivas ante interrogantes que aún no encontraron soluciones satisfactorias.

Claro que apenas con creatividad no alcanza, a esto se suma la necesidad de conocer muy bien el campo en el que se trabaja es difícil que un lego realice algún aporte importante sin conocer exhaustivamente la problemática abordada. Es importante que los tesistas e investigadores que se inician, además de conocer autores y corrientes que trabajan el tema investigado, puedan ejercer su capacidad creativa, que sean estimulados a plantear nuevas perspectivas y respuesta originales distintas a las encontradas hasta ese momento. Repetir el pensamiento de otros autores es un terreno seguro, sobre todo si se trata de autoridades reconocidas en la materia, 
pero la originalidad y el aporte creativo si bien un tanto más riesgoso, es un componente necesario de la investigación y de la producción de conocimiento científico original.

El otro punto relevante, relacionado con la justificación de hipótesis tiene que ver también, con la objetividad del conocimiento científico. Pues si bien las hipótesis precisan de creatividad e imaginación, el mero hecho de tener una idea creativa no quiere decir que se ha producido conocimiento científico. Aquí Hempel pone acento en la justificación de las conjeturas, el autor propone un esquema de prueba de tipo empírico-inductiva. Es cierto que, atendiendo a las ciencias naturales, como bien afirma Klimovsky (2005), el método inductivo-estadístico es ampliamente utilizado y aceptado. Pero ¿qué validez y utilidad tiene esto para las humanas y sociales?

Con las palabras de ciencias humanas y sociales abarcamos una variedad de disciplinas muy grande, hay algunas en que la verificación experimental de las hipótesis o conjeturas es usual y sumamente importante. En economía y sociología el uso de métodos inductivo-estadístico es generalizado y relevante. Asimismo, la recopilación de datos a través de entrevistas y/o grupos focales o métodos, así como el análisis cualitativo de los mismo es igualmente importante en áreas como educación y psicología. Sin embargo, hay disciplinas pertenecientes al área de humanidades, como la filosofía en sus diversas ramas, en que el recurso a los datos empíricos para justificar sus tesis o conjeturas es inusual. O las disciplinas ligadas a las ciencias jurídicas que por definición trabajan con lo que "debe ser", más que con lo que efectivamente "es". En general se trata de disciplinas que utilizan metodologías conceptuales y argumentativas de carácter teórico, proponen conceptos y estrategias argumentativas a fin de probar, justificar y convencer de la corrección de sus posiciones. Este artículo y, posiblemente, la mayoría de los que son publicados en revistas de filosofía define un método de justificación para sus afirmaciones que se basa en un análisis conceptual de ciertas ideas y su posible utilidad pragmática en la construcción de una tesis. No hay una prueba experimental a partir de alguna recopilación de datos empíricos sobre investigadores del área que utilicen estos conceptos. Si tomáramos la estrategia de prueba empírica la finalidad del articulo sería otra y 
posiblemente sería más adecuado para una publicación especializada en temas de educación, por ejemplo, que en filosofía.

En contraposición, si bien el recurso a la metodología empírica es poco frecuente en las disciplinas filosóficas, las discusiones filosóficas y epistemológicas son sumamente frecuentes en el área de las ciencias sociales que utilizan métodos empíricos e incluso modelos matemáticos en sus desarrollos como, por ejemplo, la economía (OLIVERA, 2010).

Y esto nos lleva a una cuestión pragmática fundamental en la elaboración de una tesis o disertación en el ámbito de las ciencias humanas y sociales: la construcción del marco teórico y metodológico. En nuestras disciplinas dedicamos una enorme cantidad de energía y horas de trabajo a la construcción de este marco, en general mucho más que las ciencias naturales. La construcción de este marco está íntimamente ligado a la delimitación del problema, la enunciación de la hipótesis y la metodología propuesta. No es que las ciencias naturales no tengan marco teórico y metodológico, sino que hay mucho menos discusión en torno de él, consecuentemente no tienen que dedicar mucho tiempo y energía en construirlo, sino que se encuentra establecido y consensuado en sus líneas fundamentales. Esta es una cuestión importante a comprender para el investigador social que se inicia. Un autor que hace un interesante aporte a fin de comprender esta problemática es Thomas Kuhn y que, si bien es una figura de la llamada Nueva Filosofía de la Ciencias inaugurada a finales de los años 1960, a esta altura podemos considerarlo un clásico del área.

\section{La construcción del marco teórico y metodológico en las tesis y disertaciones en ciencias humanas y sociales}

En el año 1958 Kuhn es invitado a participar del Center for Advanced Studies in the Behavioral Science, en Stanford. Allí compartió su estancia con científicos sociales lo que le permitió observar un contraste notable entre el comportamiento de la comunidad de científicos sociales y las de científicos naturales donde se había formado (GAETA, et al, 2007). En especial respecto al tipo y tema de discusiones y desacuerdos: 
Principalmente me asombré ante en número y el alcance de los desacuerdos patentes entre los científicos sociales, sobre la naturaleza de problemas y métodos científicos aceptados (...) la práctica de la astronomía, de la física, de la química o de la biología, no evoca, normalmente, las controversias sobre fundamentos que, en la actualidad, parecen a menudo endémicas, por ejemplo, entre los psicólogos o los sociólogos. (KUHN, 2004, p.13).

El autor sostiene que en las comunidades de científicos naturales se debate sobre temas muy específicos de sus disciplinas y que es infrecuente la discusión sobre las bases de las ciencias. Es decir, no hay desacuerdos sobre los fundamentos de la disciplina, o lo que es lo mismo, hay un consenso amplio sobre el marco teórico y metodológico: sobre la manera de plantear un problema, las teorías que permiten conceptualizarlo y las metodologías disponibles para resolverlo. Esta diferencia es crucial para entender una de las mayores diferencias entre el trabajo del científico social y el científico natural. El primero deberá dedicar una parte muy importante de su tiempo a establecer o incluso construir un marco teórico en que el su problema de investigación tenga sentido, así como una metodología acorde que permita responder el interrogante planteado. El segundo no tendrá que hacerlo pues en su comunidad científica existe un consenso generalizado sobre el mismo.

Es conocido el hecho de que en el intento de explicar esta divergencia entre las ciencias sociales y las naturales está uno de los gérmenes de la idea de paradigma que hará famoso al autor estadounidense. Básicamente sostiene que las ciencias naturales alcanzaron un período de madurez al establecer un paradigma, concepto polémico que no discutiremos, pero que implica una visión unificada de la comunidad científica acerca de los fundamentos de su ciencia: definición de objeto de estudio, marco teórico y metodológico.

La situación de las ciencias sociales es diferente, según el autor aún no alcanzaron su "madurez" y por eso es frecuente la fragmentación entre escuelas que tienen concepciones a veces radicalmente distintas del propio objeto de su ciencia, de las teorías adecuadas para abordarlo y de los métodos para hacerlo. Esta situación es notoria en las disciplinas sociales y aún más en las investigaciones filosóficas donde esta diversidad incluso es vista como algo positivo. 
Más allá del juicio de valor que implica señalar la madurez o no de un conjunto de disciplinas, lo cierto es que arduas discusiones teóricas y metodológicas deberán ser abordadas en casi todas las investigaciones sociales o de ciencias humanas. Deberá seleccionarse uno de los marcos teóricos en disputa o construir uno propio en general como variante o combinación de algunos anteriores, deberá definirse claramente el objeto de estudio, pues no todos entienden del mismo modo el objeto de su disciplina. Por ejemplo, es claro que la escuela conductista diverge radicalmente respecto a lo que debe ser estudiado por la ciencia psicológica respecto a la escuela psicoanalítica y no solo respecto a los objetos sino también a los métodos. Y no son las únicas escuelas o teorías en disputa en esa área. Podemos encontrar divergencias similares en todas las ciencias sociales y humanas. Por lo tanto, el científico social tarde o temprano tendrá que abordar las discusiones que observó con cierta sorpresa Kuhn durante el período en el que estuvo en la comunidad de científicos sociales de Stanford.

Centrándonos ahora en el objetivo específico de este trabajo que es abordar la utilidad de algunos conceptos epistemológicos en la construcción de tesis y disertaciones tenemos que preguntarnos ¿cuál es la utilidad de esta idea de Kuhn en relación de este tema? Así como el autor estadounidense percibió con algo de sorpresa el tipo de discusiones que se daban en las ciencias sociales, el investigador que está iniciándose con su tesis o disertación no siempre es consciente del grado de divergencia y polémica que existe en torno a la disciplina que aborda. Tendrá que dedicar una buena cantidad de tiempo y energía a abordar esas discusiones a fin de orientarse y posicionarse respecto a ellas, es decir: deberá construir su marco teórico y metodológico. Este es una cuestión sumamente espinosa que el investigador en ciencias naturales no necesita enfrentar, no deberá posicionarse en torno a los fundamentos mismos de su disciplina, discutir y aclarar sus conceptos fundamentales y el sentido específico que dará a los mismos. No es necesario que un físico aclare en que sentido entiende el concepto de "masa" o "velocidad", en general el biólogo no necesita aclarar que entiende por “evolución” o cuál es la definición más adecuada de "célula". Pero es sumamente frecuente que en una tesis en el área de educación se defina qué se entiende por "educación" en determinado marco teórico y se diferencie de 
otros sentidos o acepciones posibles, o que en el marco de los estudios culturales se defina cual será el sentido dado a la palabra "cultura". Y es espacio dedicado a estas definiciones no es menor, incluso hay tesis completas que se plantean solo discutir estos conceptos.

La construcción del marco teórico y metodológico es un problema sumamente complejo que el investigador social deberá enfrentar y representa un enorme esfuerzo para quién se inicia, pues deberá abordar, aunque sea parcialmente, alguno de las cuestiones más complejas y fundamentales de su disciplina. Tener consciencia de este desafío y de la complejidad de esta tarea, de la necesidad de recorrer estas discusiones y de orientarse es ellas a fin de tomar una posición propia es un paso fundamental de la investigación social. Comprender la situación de división en escuelas, de que muchas veces los conceptos principales de un área tienen sentidos radicalmente diferentes en un abordaje respecto de otro, es sumamente importante para la tarea de investigación. Es usual que el pesquisador novel sienta cierta desorientación ante la dificultad y la envergadura de la tarea que enfrenta, los problemas parecen al principio inabarcables. Tener conciencia de ello, saber en lo que la tarea consiste y la necesidad de realizarla, disminuye el sentimiento de desorientación, focaliza la tarea y el esfuerzo y permite comprender que, aún la posición mejor fundamentada y argumentada, siempre será plausible de objeciones y críticas.

La definición del marco teórico y metodológico, así como la especificación y definición del sentido de los términos principales que utiliza el investigador, está estrechamente relacionado al punto anterior: la definición del problema a investigar y la propuesta de hipótesis para resolverlo. Esta relación consiste en que muchas veces plantear un problema dentro de un marco teórico, tiene sentido en ese marco, pero no en otro de la misma disciplina. Por ejemplo, plantear algún problema de investigación que envuelva el concepto de "inconsciente" tendrá sentido dentro de un abordaje psicoanalítico de la psicología, pero no lo tendrá dentro del marco teórico y metodológico de la escuela conductista, e incluso dentro de la escuela psicoanalítica la idea de "inconsciente colectivo" será aceptable para alguna escuela, pero no para otros enfoques. Del mismo modo el concepto de "plusvalía" o de "alienación" serán relevantes en los problemas planteados 
desde un análisis económico dentro del marco teórico marxista, pero no serán admisibles dentro de un abordaje económico enmarcado en las teóricas neoclásicas o neoliberales.

Es decir, el planteo correcto de un problema e incluso el sentido de este dependerá del marco teórico seleccionado o construido, pudiendo incluso no ser considerado un problema relevante dentro de otro marco. Lo mismo ocurrirá con las posibles respuestas a estos problemas, es decir las hipótesis, en el sentido de que muchas serán consideradas respuestas válidas y adecuadas dentro de un enfoque teórico, pero no en otro.

Pero ¿acaso sucede lo mismo con las ciencias naturales? En ellas también los problemas relevantes y las hipótesis aceptables parecen determinadas por el marco teórico. Es cierto, todos los científicos trabajan bajo un marco teórico y metodológico que proporciona el fundamento para poder plantear problemas e intentar encontrar soluciones. La diferencia es que en estas disciplinas el marco teórico se encuentra unificado, como bien señala Kuhn hay pocos debates relevantes sobre las bases teóricas de las disciplinas y sus conceptos fundamentales. Se presentan bien definidos y son aceptados por las comunidades científicas con poca discusión. Esto hace que el científico natural dedique poco o ningún tiempo y energía a debates sobre marcos teóricos, metodológicos y definiciones de conceptos fundamentales. Se dedican a problemas también complejos, pero enunciados dentro de un marco común a toda la disciplina.

Esta es una diferencia relevante en la práctica científica entre las ciencias humanas y sociales y las ciencias naturales. Es importante ser conscientes de esta circunstancia al momento de elaborar un proyecto de investigación en nuestra gran área.

Para finalizar señalaremos una situación curiosa respecto a la recepción del pensamiento de Kuhn dentro de las ciencias sociales. La recepción en el área fue paradójicamente positiva (GAETA, et al, 2007) aun cuando el autor afirma la inmadurez de esta al no poder consolidar una visión unificada de sus propias disciplinas. Gaeta y Gentile señalan que esto se debe a que, más allá de esa visión crítica sobre las ciencias sociales, el autor también propone una visión desmitificadora respecto de ciencias duras cómo física o astronomía en las que se a formado, pues platea serias dudas sobre la 
exactitud, perfección y objetividad de sus métodos. Esto indirectamente contrarresta la visión de superioridad de estas ciencias naturales sobre las ciencias sociales, fundamentalmente en la visión clásica de Popper o el círculo e Viena que niegan el estatus científico de muchas disciplinas y teorías sociales.

Concuerdo con esas razones, pero creo que existe otra razón más poderosa que justifica la recepción positiva de la epistemología kuhniana en nuestra área. El propio Kuhn en sus tesis utiliza conceptos provenientes de las ciencias sociales, construye un marco teórico diferente del utilizado por las escuelas epistemológicas anteriores y genera una gran polémica discutiendo los fundamentos mismos de la epistemológica clásica: la centralidad del análisis lógico y empírico o la distinción entre contexto de descubrimiento y contexto de justificación. El autor propone un marco teórico/conceptual novedoso y se convierte en uno de los representantes de la llamada Nueva Filosofía de la Ciencia:

\begin{abstract}
Durante aquellos años, tomó además conocimiento de trabajos sobre psicología de la percepción -especialmente los textos de los teóricos de la Gestald- y de las investigaciones de Jean Piaget sobre el desarrollo de la inteligencia del niño. Se interesó, asimismo, en el estudio de los escritos de Whorf acerca del lenguaje sobre la percepción del mundo y en las especulaciones de Quine referidas a los problemas relativos a la distinción analítico-sintética. La consideración de todos estos aspectos, junto con sus investigaciones en la historia de la ciencia, le llevaron a adoptar un particular punto de vista acerca de la actividad científica y de la evolución de la ciencia, diametralmente diferente de la concepción tradicional. (GAETA, et al, 2007, p.129)
\end{abstract}

Lo que describen los autores en el párrafo citado es justamente el derrotero que recorrió el autor a fin de construir su propio marco teórico original y novedosos que incluiría nuevos conceptos y definiciones a fin de analizar el objeto clásico de la epistemología: el conocimiento científico que definirá, por supuesto, de manera nueva y diferente. La concepción de ciencia expresada por Kuhn en sus escritos más famosos es radicalmente distinta a la de los autores tradicionales, es decir hay una discordancia en los propios conceptos fundamentales de la disciplina, una discusión que recuerda a las apuntadas por Kuhn como típica de las ciencias sociales.

En pocas palabras Kuhn un físico formado en la comunidad científica de ciencias duras, cuando intenta comprender y conceptualizar su propia 
actividad recurre a la estrategia discursiva y al método propio de las ciencias sociales: construye su propio marco teórico con aportes de varios autores que considera relevantes, discute con las concepciones anteriores y presenta un punto de vista nuevo, distinto y original. La propia práctica del autor legitima a las ciencias sociales. Si bien teóricamente parece criticarlas, su práctica las legitima mostrando que tienen valor en tanto contribuyen a la comprensión rigurosa de su objeto de conocimiento. No es extraño entonces que sus trabajos tengan una buena recepción en el ámbito de la ciencias sociales y humanas, pues su propia práctica las muestra como una forma legítima de producir conocimiento.

\section{Conclusiones}

La primera conclusión que podemos extraer de la serie de argumentaciones presentadas en este artículo, es que hemos alcanzado el objetivo propuesto: seleccionado alguno de los principales conceptos discutidos por la epistemología durante el siglo veinte, es decir autores ya considerados clásicos, y los hemos analizado en función de la utilidad que pueden tener en la tarea práctica de la construcción de una tesis o disertación en el área de las ciencias sociales y humanas. En este apartado entonces recopilaremos las conclusiones parciales de cada capítulo a fin de fundamentar nuestra conclusión final.

El primer concepto que postulamos como útil es la idea de construir una tesis atendiendo a que se trata un razonamiento o argumento complejo. Más allá de que el proceso de investigación real no sea siempre tan lineal $\mathrm{u}$ ordenado, al momento de construir su producto final, de expresar el resultado de la investigación, debemos hacerlo en la forma de un argumento. En el sentido de presentar una serie de argumentos lógicamente conectados que, a partir de una sucesión de datos e inferencias, justifiquen y fundamenten la tesis que sostenemos. En general cada capítulo de una tesis es parte de la fundamentación de la idea principal que será explicitada y justificada finalmente en las conclusiones.

Luego tomamos las ideas de Hempel que sostienen que una investigación comienza con un problema científico bien plateado, una o varias hi- 
pótesis que dan respuesta al problema y que deben ser evaluadas y ajustadas durante el proceso de investigación. Estos conceptos son importantes por su valor intrínseco en tanto sirven para guiar el proceso, a veces un tanto azaroso, de una investigación. Pero también tienen una utilidad pragmática pues están implícitos en los requerimientos de las agencias científicas al momento de postular un proyecto de investigación: enunciar el objetivo del proyecto, el problema que se tratará, la hipótesis con la que se pretende abordar el problema, y la metodología para probarla. Aquí comenzamos a percibir que el marco teórico y metodológico que permite plantear un problema y ofrecer respuestas en forma de hipótesis, funciona de manera diferente en las ciencias sociales y humanas que en las naturales. Esto nos lleva al próximo concepto epistemológicamente interesante.

La tercera idea evaluada es la que Kuhn llama fragmentación de escuelas: en las comunidades de científicos sociales existen discusiones sobre temas fundantes de las disciplinas casi completamente ausentes en las comunidades de científicos naturales. Las diferentes escuelas discuten sobre la propia definición de la disciplina, sobre el objeto de estudio a abordar y la legitimidad de los métodos para hacerlo. Estas polémicas están prácticamente ausentes en las comunidades de científicos naturales donde existe, en cambio, un consenso amplio sobre esas cuestiones lo que implica la existencia de un marco teórico y metodológico unificado.

Esta situación hace que el investigador social tenga que dedicar una parte importante de su tiempo y energía a estas discusiones: desde el inicio deberá enfrentar la tarea de elegir el marco adecuado entre los varios en disputa o construir el suyo propio. Esta tarea es de suma complejidad y significa un enorme esfuerzo para quien se inicia pues implica enfrentar desde estas primeras etapas algunos de los problemas más complejos de su disciplina. Ser consciente del desafío que plantean las ciencias sociales y de la complejidad de las cuestiones que el investigador novel debe enfrentar, es útil para dimensionar el proyecto de investigación a realizar, para no desalentarse ante estos desafíos y para poder culminar investigaciones con éxito. Y es importante también para comprender los límites de nuestras disciplinas: aún la tesis mejor fundamentada es susceptible de objeciones y cuestionamientos especialmente desde marcos teóricos alternativos al elegido. 
El concepto crítico enunciado por Kuhn como un rasgo de inmadurez de las ciencias sociales, parece sin embargo no deslegitimar el conocimiento producido por las mismas. Pues el propio autor, físico devenido epistemólogo, a fin de comprender su propia actividad científica, construye su propio marco teórico de manera original a fin de cuestionar y polemizar con escuelas epistemológicas anteriores. Esto le permite brindar una nueva perspectiva sobre su nueva área de conocimiento. Kuhn procede como un científico social para explicar qué son las ciencias naturales. Esto implica reconocer en la práctica el valor de las ciencias sociales y humanas para comprender e interpretar sus complejos objetos de estudio: los seres humanos y su comportamiento en sociedad. Comportamiento que incluye, por supuesto, la producción de conocimiento científico.

\section{Referencias}

BLASCO, J. Método analítico y transcendentalidad. Revista filosófica, vol. IX, n. 16, p. 41-56, 1996.

BUENO, G. En torno al concepto de ciencias humanas. El Basilisco, año 1, vol 2, p. 12-47, 1978. Disponible en:http://fgbueno.es/bas/bas10202.htm. Acceso en: 27 de junio 2020.

COPI, I. Introducción a la lógica. Buenos Aires: Eudeba, 1992.

COPI, I.; COHEN, C. Introducción a la lógica. México: Limusa, 2013.

DE LUQUE, S. La problemática valorativa-metodológica en las ciencias sociales. En: Diaz, E. (comp) Metodología de las ciencias sociales. Buenos Aires: Biblos, 2010.

HEMPEL, C. Filosofía de la ciencia natural. Madrid: Alianza, 1983.

GAETA, R.; ROBLES, N. Nociones de epistemología. Buenos Aires: Eudeba, 1990.

GAETA, R.; GENTILE, N. Thomas Kuhn: de los paradigmas a la teoría evolucionista. Buenos Aires: Eudeba, 2007.

GIANELlA, A. Lógica Simbólica Y Elementos De Metodología De La Ciencia. Buenos Aires: Ed. Cooperativa, 2002.

KLIMOVSKY, G. Las desventuras del conocimiento cientifico. Buenos Aires: A-Z Editora, 2005. 
KUHN, T. La estructura de las revoluciones científicas. México: FCE, 2004.

MIRÓ QUESADA, F. La lógica paraconsistente y el problema de la racionalidad de la lógica. En MIRO QUESADA F.; CARRION, R.; (eds). Antología de la lógica en América Latina. Madrid: Fundación Banco Exterior, p. 593-622, 1988.

OLIVERA, J. La ciencia económica como una hermenéutica social. Conferencia de apertura de la XLV Reunión Anual de la Asociación Argentina de Economía Política, Buenos Aires, 17 de noviembre de 2010. Disponible en: https://aaep.org.ar/anales/download/2010/OliveraANCE2010.pdf. Acceso en: 27 de junio 2020.

POPPER, K. La lógica de la investigación científica. Madrid: Tecnos, 1980.

RUSSELL, B. Introducción a la filosofía matemática filosófica. Madrid: Aguilar, 1956. 